\title{
Una década de investigación universitaria sobre Comunicación en España, 2007-2018
}

\section{A decade of scholarly research on Communication in Spain (2007-2018)}

\author{
Carlos Lozano-Ascencio; Juan-Antonio Gaitán-Moya; Carmen Caffarel-Serra; \\ José-Luis Piñuel-Raigada
}

Cómo citar este artículo:

Lozano-Ascencio, Carlos; Gaitán-Moya, Juan-Antonio; Caffarel-Serra, Carmen; Piñuel-Raigada, José-Luis (2020). "Una década de investigación universitaria sobre Comunicación en España, 2007-2018". Profesional de la información, v. 29, n. 4, e290412.

https://doi.org/10.3145/epi.2020.jul.12

Artículo recibido el 10-01-2020 Aceptación definitiva: 05-05-2020

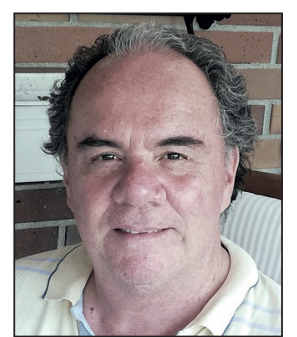

Carlos Lozano-Ascencio https://orcid.org/0000-0003-2537-0799

Universidad Rey Juan Carlos

Facultad de Ciencias de la Comunicación Camino del Molino, 5.

28943 Fuenlabrada (Madrid), España carlos.lozano@urjc.es

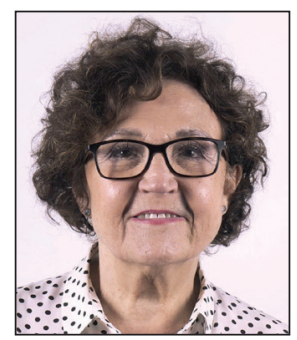

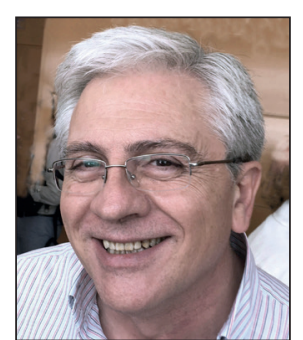

Juan-Antonio Gaitán-Moya https://orcid.org/0000-0002-4496-7315

Universidad Complutense de Madrid Facultad de Ciencias de la Información Av. Complutense, 3, 28040 Madrid, España jagamo@ucm.es

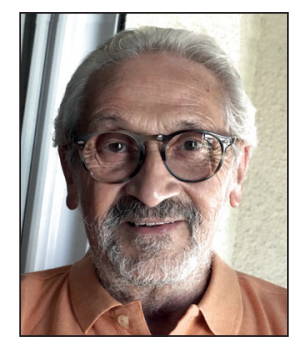

José-Luis Piñuel-Raigada https://orcid.org/0000-0003-1354-0770

Profesor honorífico

Universidad Complutense de Madrid Facultad de Ciencias de la Información Av. Complutense, 3, 28040 Madrid, España pinuel@ucm.es

\section{Resumen}

Se analizan los resultados obtenidos entre 2007 y 2018 del cómputo total de, 1) tesis doctorales defendidas en las 55 universidades con Facultades de grados y/o postgrados en comunicación; 2) y en éstas, los proyectos competitivos en convocatorias estatales en Ciencias Sociales y Humanidades y cuyo objeto de estudio sea una dimensión socialmente relevante de las prácticas de "Comunicación"; y 3) artículos derivados de estos proyectos I+D publicados en las revistas de referencia en Comunicación. Estos documentos son los más representativos de la actividad investigadora de los profesores universitarios, y constituyen un censo de publicaciones basadas en investigaciones previas. Los artículos cuyo objeto de estudio no ha sido una investigación previa no han sido seleccionados (ensayos, revisiones epistemológicas de autores, escuelas y/o tendencias teóricas o metodológicas). Las comunicaciones presentadas en congresos de la Asociación Española de Investigación de la Comunicación $(A E-I C)$ sólo son consideradas en esta exposición de forma tangencial, pues sólo una minoría de ellas es resultado de investigaciones originales. Con tales resultados se ha perfilado mediante un análisis comparativo de contenido, la productividad de la investigación en Comunicación en España durante una década, tomando en consideración los respectivos censos de documentos referidos según universidades, y que supone el análisis de Comunidades autónomas, género de sus autores y años. Se ha analizado un total de 4.158 documentos. Los resultados permiten contemplar, como conclusión más general, una evolución histórica marcada por la influencia de las políticas científicas derivadas de la imposición de las agencias de evaluación de la calidad investigadora.

\section{Palabras clave}

Comunicación; Metainvestigación en Comunicación; Universidad española; Tesis doctorales; Proyectos I+D; Artículos científicos; Ponencias; Comunicaciones en congresos. 


\begin{abstract}
We analyzed the results obtained for the total count between 2007 and 2018 of (1) doctoral theses defended in the 55 universities with undergraduate and/or graduate studies in communication that offer doctoral degrees, (2) competitive projects in state calls in the social sciences and humanities areas where the object of study includes a socially significant dimension of "communication" practices, and (3) papers resulting from R\&D projects published in reference journals in the area of communication. These documents are the most representative of the research activity of faculty, because they constitute the published results originating from research that is recognized and legitimized by academic life, without which such documents could not have been published. Thus, the articles collected here constitute a census of publications based on previous research, since all those articles whose object of study was not previous research were not selected, e.g., essays, or epistemological reviews of authors, schools, and/or theoretical or methodological trends. Papers presented at Asociación Española de Investigación de la Comunicación $(A E-I C)$ congresses, which are also being studied as part of the MapCom project, could also have been included; however, they are only considered tangentially herein because only a minority of them are expressly linked to research results. This overview of the productivity of communication research in Spain over a decade analyzes documents grouped by universities, Autonomous Communities, author gender, and years. A total of 4,158 documents have been analyzed. The results reflect the influence of the scientific policies of the research quality assessment agencies.
\end{abstract}

\title{
Keywords
}

Communication; Metaresearch in communication; Spanish universities; Doctoral theses; R\&D projects; Scholarly articles; Papers; Conference proceedings.

\section{Introducción y objeto de la investigación}

Conocer el sistema de investigación en comunicación en España permite planificar políticas públicas de investigación y mostrar a los investigadores del área el estado de la cuestión para recabar nichos de interés de futuros trabajos según qué objetos, qué métodos, qué técnicas, qué fines son los más estudiados y desde qué perspectivas teóricas. Tanto en la primera como en la segunda etapa de MapCom (proyectos que sustentan este trabajo www.mapcom.es) se aspira a ir más allá del mero repositorio de documentos (como Teseo para las tesis doctorales) elaborando una cartografía que representa datos del análisis de los documentos producidos por la investigación. Se analizaron las tesis doctorales y los proyectos I+D+l entre 2007 y 2013 en el primer proyecto MapCom, y en el segundo proyecto, a las tesis doctorales y proyectos competitivos I+D+l entre el 2014 y 2018 se añadieron las publicaciones de la década en revistas científicas y en libros de editoriales de referencia, así como las comunicaciones aportadas a los congresos de la $A E-I C$ desde sus orígenes hasta 2018. En este artículo se ofrecen los primeros resultados del análisis longitudinal de las tesis doctorales, los proyectos I+D+I, las publicaciones en revistas y las comunicaciones en congresos, durante la década, según universidades, comunidades autónomas (CC. AA.), y género de sus autores.

El interés por la metacomunicación se remonta a la década de los noventa del pasado siglo con investigaciones auspiciadas, muchas de ellas, por la primitiva AICE (Asociación de Investigadores en Comunicación del Estado Español) entre las que destacan Caffarel-Serra, Domínguez y Romano (1989), Cáceres-Zapatero y Caffarel-Serra (1993), y Jones (1994, 1998) quienes analizaron el estado de la investigación en Comunicación en las facultades de Ciencias de la Información en su vigésimo aniversario, Facultades herederas de las antiguas escuelas profesionales de periodismo, imagen y publicidad y por tanto más orientadas al ejercicio profesional que al conocimiento científico de una realidad, la comunicación, que impregnaba ya todos los aspectos de la vida social. Recientemente, el interés por la metainvestigación en comunicación ha vuelto a cobrar fuerza. Entre otros, destacamos los trabajos de Rodrigo-Alsina y García-Jiménez (2011); Piñuel-Raigada (2011); Lozano-Ascencio y Gaitán-Moya (2011); Fernández-Quijada y Masip (2013); Arcila-Calderón, Piñuel-Raigada y Calderín-Cruz (2013); trabajos que vuelven a coincidir con otra efeméride, el quincuagésimo aniversario de las facultades pioneras de Ciencias de la Información.

Posteriormente, otros proyectos de investigación como el recientemente citado MapCom "El sistema de investigación en España sobre prácticas sociales de Comunicación. Mapa de proyectos, líneas, objetos de estudio y métodos" liderado por José-Luis Piñuel-Raigada han aportado valores fundamentales tales como su política de datos abiertos (Caffarel-Serra et al., 2019a; 2919b), para generar conocimiento y dar a conocer a la comunidad científica, a sus dirigentes y a quienes han de diseñar las políticas públicas de investigación, cuál es el panorama de esa investigación que auspician y subvencionan.

Hay también antecedentes de investigadores nacionales que se han ocupado de forma parcial del objeto de estudio. Entre otros, estudios sobre

- la historia de la investigación en comunicación: Martínez-Nicolás (2009); Vidales-Gonzales (2015);

- epistemología y metodología de la metainvestigación: Fuentes-Pujol (1994); Giménez-Toledo y Jiménez-Contreras (2013); Vasallo (1999); Piñuel-Raigada et al. (2015); Gaitán-Moya et al. (2018); Rodríguez-Serrano y Gil-Soldevilla (2018).

- opinión que reflejan los investigadores en comunicación: Piñuel (2009; 2011; 2015); Gaitán-Moya (2014); Lozano-Ascencio y Gaitán-Moya (2016); Caffarel-Serra et al. (2018).

También hay precedentes sobre documentos del mismo género que vamos a considerar en este artículo: 
- tesis doctorales: Delgado-López-Cózar et al. (2006); Castillo-Esparcia y Xifra (2006); Blázquez-Ochando (2015); Fuentes-Pujol y Arguimbau-Vivó (2010); Repiso-Caballero et al. (2011);

- tesis doctorales y proyectos I+D: Caffarel-Serra et al. (2018b); Martín-Algarra et al. (2018); Caffarel-Serra et al. (2017); Díaz Nosty y De-Frutos-García (2016); Gaitán-Moya et al. (2016); Barranquero-Carretero y Limón-Serrano (2017); López-Escobar y Martín-Algarra (2016);

- artículos y libros científicos: Masip (2005); Castillo-Esparcia y Carretón-Ballester (2010); Martínez-Nicolás y Saperas (2011); Fernández-Quijada y Masip (2013); De-Filippo (2013); y

- comunicaciones presentadas a congresos de Comunicación de la AE-IC; Rodríguez-Gómez (2016).

Por último, hay diversas referencias, trasversales, a propósito de la infrapresencia de la mujer en el ámbito de la investigación sobre comunicación contraviniendo las recomendaciones, especialmente la 3a , del Parlamento Europeo en su programa Horizonte 2020 (Official Journal of the European Union, 2013).

Sin embargo, es la primera vez que se aborda una investigación sobre el conjunto de documentos que caracterizan la productividad investigadora.

\section{Metodología}

La primera fase del proyecto MapCom en su segunda etapa, ha consistido en hacer un repositorio de los documentos que simbolizan la labor investigadora empezando por las tesis doctorales (en adelante TD), proyectos de investigación I+D+l españoles (en adelante $\mathrm{PI}+\mathrm{D}$ ), publicaciones en revistas científicas en el área de comunicación, libros publicados en las editoriales de mayor prestigio, y finalmente comunicaciones a congresos, en un marco temporal que abarca desde 2007 a 2018.

Además, se añade un repositorio de los grupos de investigación en Comunicación consolidados en las universidades que ofrecen grados o postgrados en Comunicación. De los 6 repositorios obtenidos, en este trabajo vamos a explotar los resultados a partir de los tres primeros: TD, PI+D y artículos científicos. Estos se corresponden con la trayectoria de la actividad investigadora; las TD suponen el inicio al mundo de la investigación, los PI+D suponen el reconocimiento por parte de las instituciones que regulan las políticas científicas; y los artículos son el mejor instrumento para la transferencia del conocimiento, por eso hemos incluido en este estudio aquellos en los que expresamente se hace referencia a un proyecto originario de investigación. Respecto a las comunicaciones de congresos de la $A E-I C$ solo se tendrán en cuenta en esta exposición para compararlas con los otros documentos, por comunidad autónoma y universidades.

En el caso de las TD y PI+D contábamos con el censo desde 2007 a 2013 resultado del proyecto MapCom1 en el que obtuvimos un universo de 979 TD y 143 PI+D. Se realizó una búsqueda de las TD y PI+D desde 2014 a 2018 con los mismos criterios; para las tesis doctorales, a partir de la base Teseo:

https://cutt.ly/yraRXE8

y reseñamos las tesis defendidas en las universidades españolas, públicas y privadas, que tuvieran grados o postgrados en Comunicación. El resultado fue de 1.598 documentos que, sumados a los 979 que se obtuvieron de 2007 a 2013 , hacen un total de 2.577 tesis doctorales.

Para los PI+D el Ministerio nos facilitó el código de referencia, la universidad, título del proyecto, el año de concesión, la duración temporal y la financiación; sin embargo, no nos facilitó el/los nombre/s del investigador principal, así como tampoco el resumen ejecutivo que figura, como publicable, en la memoria científica de los expedientes y que también le habíamos solicitado. Por ello escribimos a los vicerrectores de investigación de las universidades a las que se le habían otorgado los proyectos, solicitándoles esa información. Hubo dos tipos de respuestas: una, desde los vicerrectorados nos facilitaron la información solicitada y, otra, los vicerrectorados se pusieron en contacto con los investigadores principales (IPS) quienes nos facilitaron los datos.

El resultado de PI+D obtenidos fue de 172, que sumados a los 143 proyectos analizados desde 2007-2013 conforman un universo de $315 \mathrm{PI}+\mathrm{D}$.

Para elaborar el censo de artículos publicados en revistas desde 2007 a 2018 se decidió registrar aquellos publicados en las primeras revistas españolas según el índice h, elaborado por Google Scholar, especializadas en Comunicación (Delgado-López-Cózar et al., 2013; Delgado-López-Cózar y Martín-Martín, 2018). En el periodo 2013-2017 aparecen las revistas listadas en la tabla 1.

Tabla 1. Índice h de las revistas científicas españolas de comunicación de mayor impacto según Google Scholar Metrics 2013-2017

\begin{tabular}{|c|c|c|}
\hline Revistas & Índice $h$ & Mediana $\mathbf{h}$ \\
\hline Comunicar. Revista científica iberoamericana de comunicación y educación & 38 & 50 \\
\hline Profesional de la información & 22 & 31 \\
\hline Revista latina de comunicación social & 18 & 24 \\
\hline Comunicación y sociedad & 17 & 27 \\
\hline Historia y comunicación social & 15 & 19 \\
\hline Estudios sobre el mensaje periodístico & 14 & 23 \\
\hline
\end{tabular}

Fuente: Delgado-López-Cózar y Martín-Martín (2018) 
Como el periodo que teníamos que analizar arrancaba desde el año 2007, buscamos las revistas mejor indexadas de ese periodo (tabla 2).

Tabla 2. Índice h de las revistas científicas españolas de comunicación de mayor impacto según Google Scholar Metrics 2007-2011

\begin{tabular}{|c|c|c|}
\hline Revistas & Índice $h$ & Mediana h \\
\hline Telos: Cuadernos de comunicación e innovación & 10 & 13 \\
\hline Revista Latina de comunicación social & 9 & 17 \\
\hline Comunicar: Revista científica iberoamericana de comunicación y educación & 9 & 14 \\
\hline Zer: Revista de estudios de comunicación = Komunikazio ikasketen aldizkaria & 7 & 12 \\
\hline Estudios sobre el mensaje periodístico & 7 & 8 \\
\hline Comunicación y sociedad & 6 & 7 \\
\hline Trípodos: revista digital de comunicació & 6 & 7 \\
\hline
\end{tabular}

Fuente: Delgado-López-Cózar et al. (2013)

Teniendo en cuenta que algunas revistas se repetían optamos por analizar las diez primeras revistas, que se muestran en la tabla 3 con las cantidades de artículos que publicaron. Entre 2007 y 2018 identificamos 4.893 artículos.

Tabla 3. Número de artículos en revistas españolas de comunicación mejor indexadas en la década (2007-2018)

\begin{tabular}{|l|c|}
\hline \multicolumn{1}{|c|}{ Revista } & No de artículos \\
\hline Profesional de la información & 1.039 \\
\hline Estudios sobre el mensaje periodístico & 942 \\
\hline Telos & 715 \\
\hline Comunicar & 577 \\
\hline Revista latina de comunicación social & 557 \\
\hline Zer & 309 \\
\hline Trípodos & 305 \\
\hline Historia y comunicación social & 228 \\
\hline Comunicación y sociedad & 224 \\
\hline Total & 4.893 \\
\hline
\end{tabular}

Se seleccionaron después los artículos que se derivaban de PI+D, referenciados en el texto y sólo aquellos artículos cuyo texto completo se hallaba en abierto. Así obtuvimos un total de 413 artículos de 2007 a 2018.

Las comunicaciones presentadas a congresos solo fueron tomadas en consideración para examinar al final los perfiles de conjunto siguiendo el volumen de su evolución histórica bienal, supuesto el interés de ver esta evolución por la participación académica de las universidades en los congresos, comparada con la actividad investigadora que también, según bienios, es referida al volumen de TD, PI+D y artículos. La comparación según bienios está motivada por el hecho de que los congresos de la $A E-I C$ son bienales. Concretamente fueron elegidos los publicados en actas de los congresos organizados por la $A E-I C$ desde sus inicios ya que, en los congresos plenarios de esta Asociación, la única a nivel estatal que reúne a los investigadores en Comunicación, están representadas todas las secciones temáticas; por tanto, cubren el total de las líneas de investigación (Santiago 2008, Málaga 2010, Tarragona 2012, Bilbao 2014, Madrid 2016 y Salamanca 2018). El universo fueron 853 comunicaciones seleccionadas a partir del único criterio de que alguno de los autores perteneciera a una universidad española, ya que el objetivo de este trabajo es examinar la investigación en comunicación en las universidades españolas. Localizados todos los documentos se procedió a su vaciado y análisis de contenido seleccionando las variables de bienio, nombre completo del primer autor/es, género, universidad, comunidad autónoma, título del documento y url, palabras clave e idioma, y el nombre del congreso, así como en el caso de los artículos el nombre de la revista y en las comunicaciones el nombre del congreso y en ambos casos el número del PI+D del que derivaba la comunicación o el artículo.. Para asegurar la convergencia entre analistas se han desarrollado exprofeso sesiones y se han efectuado controles de revisión de la cumplimentación de los criterios de análisis establecidos para garantizar la denominada fiabilidad objetiva inter-jueces.

Finalmente hemos obtenido un total de 4.158 documentos que a continuación pasamos a analizar longitudinalmente en el periodo seleccionado.

\section{Resultados}

Para el análisis de los datos se seguirá un orden que permitirá comparar cada uno de los corpus citados de documentos fijando la atención en las variables siguientes: evolución temporal de los años 2007 al 2018, universidades y CC. AA. y las diferencias por identidad de género de investigadores firmantes, tal y como recomienda el programa Horizonte 2020 que en su tercer objetivo señala la incorporación de la dimensión de género en la investigación y la innovación de contenidos (Horizonte 2020). 


\subsection{Análisis de la evolución temporal de las TD, los PI+D y los artículos científicos}

Las tesis doctorales, documentos que justifican la culminación del máximo nivel que se puede aspirar en la titulación universitaria, son indispensables para iniciar una carrera académica y también son representativas de una parte muy significativa de la investigación universitaria en Comunicación. Las TD hacen posible la convivencia entre las primeras propuestas de los investigadores junior y la experiencia y guía de los investigadores senior.

En España hay 55 universidades que imparten grados y postgrados en Comunicación repartidas en 15 CC. AA. (quedan fuera Asturias y Cantabria). Se han registrado 48 universidades $(87,2 \%)$ en las que se han presentado TD entre septiembre de 2007 y diciembre de 2018 (cf. tabla 4).

Tabla 4. Acrónimos de las universidades analizadas

\begin{tabular}{|c|c|c|c|}
\hline IEU & IE University & ULL & Universidad de La Laguna \\
\hline$U A$ & Universidad de Alicante & UM & Universidad de Murcia \\
\hline$U A B$ & Universitat Autònoma de Barcelona & UMA & Universidad de Málaga \\
\hline UAN & Universidad Antonio de Nebrija & $U M H$ & Universidad Miguel Hernández \\
\hline UAOCEU & Universitat Abat Oliba & UNAV & Universidad de Navarra \\
\hline$U B U$ & Universidad de Burgos & UNIR & Universidad Internacional de la Rioja \\
\hline UC3M & Universidad Carlos III Madrid & UNIZAR & Universidad de Zaragoza \\
\hline$U C A$ & Universidad de Cádiz & UOC & Universitat Oberta de Catalunya \\
\hline UCAM & Universidad Católica de Murcia & UPF & Universitat Pompeu Fabra \\
\hline UCJC & Universidad Camilo José Cela & UPSA & Universidad Pontificia de Salamanca \\
\hline UCLM & Universidad de Castilla la Mancha & UPV & Universidad Politécnica de Valencia \\
\hline UCM & Universidad Complutense de Madrid & $U P V / E H U$ & Universidad del País Vasco/Euskal Herriko Unibertsitatea \\
\hline UCHCEU & Universidad Cardenal Herrera & URJC & Universidad Rey Juan Carlos \\
\hline$U D C$ & Universidade da Coruña & $U R L$ & Universitat Ramon Llull \\
\hline UDEUSTO & Universidad de Deusto & URV & Universitat Rovira i Virgili \\
\hline UDG & Universitat de Girona & US & Universidad de Sevilla \\
\hline$U D L$ & Universitat de Lleida & USAL & Universidad de Salamanca \\
\hline UEM & Universidad Europea de Madrid & USC & Universidade Santiago de Compostela \\
\hline UEX & Universidad de Extremadura & USJ & Universidad San Jorge \\
\hline UGR & Universidad de Granada & USPCEU & Universidad San Pablo CEU \\
\hline UHU & Universidad de Huelva & UV & Universidad de Valencia \\
\hline UIB & Universitat de les IIles Balears & UVA & Universidad de Valladolid \\
\hline UIC & Universitat Internacional de Catalunya & UVIC & Universitat de Vic \\
\hline 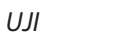 & Universitat Jaume I & UVIGO & Universiade de Vigo \\
\hline
\end{tabular}

Casi una de cada cuatro $(23,6 \%)$ de las TD en Comunicación presentadas durante los últimos once años en España se defendieron en 2016 (gráfico 1). La principal razón que explica esta circunstancia es el cambio de la normativa que regulaba los cursos de doctorado en las universidades españolas (Plan Bolonia, RD 99/2011 o "decreto Gabilondo") y que provocó un aluvión de defensas de TD, porque limitaba a cinco años (a partir de 2011) el plazo para presentar las tesis.

La acumulación de tesis registradas en el año 2016, motivada por presiones administrativas, no académicas, disparó a más de 600 los números habituales de lecturas defendidas. En el 2015 y en el 2017, por un "efecto contagio", se presentaron poco más de la mitad de TD que en el año 2016 (350). El resto de los años, 2008, 2009, 2010, 2011, 2012, 2013, 2014 y 2018 registraron entre 100 y 200 TD, es decir, en torno a la tercera parte del año 2016 (cfr. gráfico 1).

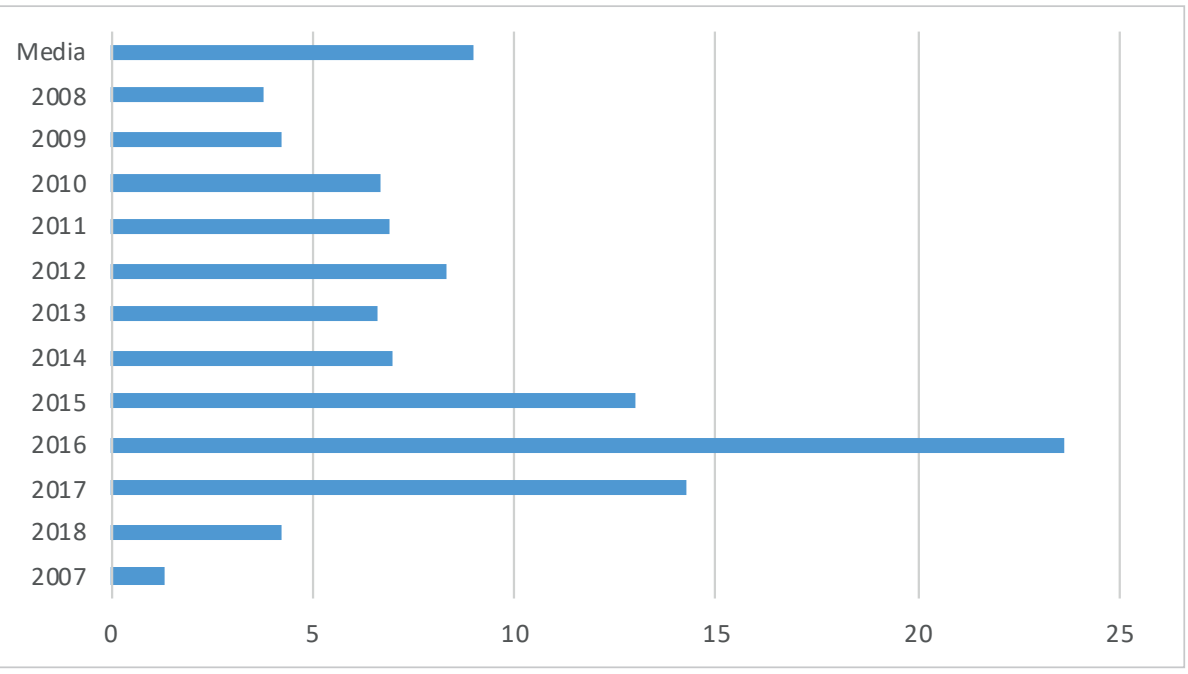

Gráfico 1. Porcentaje de distribución de tesis doctorales por años 
Si analizamos las universidades veremos que sólo 10 (la quinta parte) reflejan trayectorias similares con relación con el boom de TD en 2016. Es decir, ese año se defendieron al menos la cuarta parte del número total de sus tesis y además superando al menos el doble de las tesis defendidas otros años. Particularmente esto se observa en las universidades UA, UBU, UCM, UGR, UHU, UMA, URL, USC, UV y UVIC.

Por su parte los PI+D de convocatorias estatales aportan un mayor prestigio en la labor investigadora, ya que mediante evaluación por pares a través de la Agencia Estatal de Investigación, se compite por el financiamiento de los gastos que los proyectos seleccionados le cuestan a las universidades.

La concesión de los PI+D en estos años estudiados según se muestra en el gráfico 2 .

Se constata una peculiaridad: comprensiblemente los peores años tras la crisis (2009-2011) disminuye la media de concesiones que luego se remonta en 2012, año a partir del cual se pre-

cipita hasta llegar a los valores anteriores a la década que se estudia. El descenso relativo desde 2014 podría explicarse también por la presencia de otras convocatorias (autonómicas y de otros tipos) a las que se acogen los investigadores, sustituyendo a aquellas que hubieran optado con éxito al plan estatal antes de esa fecha.

Los artículos científicos, por su parte (cfr. gráfico 3) podrían representar un índice del rendimiento de la investigación en lo que atañe a la transferencia social del conocimiento a la que contribuyen, y esta transferencia no sería posible sin poner a disposición de la comunidad científica los resultados de la actividad investigadora, gracias a su publicación en revistas de impacto. Este encadenamiento de trasferencias puede ser medido por el recuento de artículos científicos derivados de PI+D. También constituye un índice del rendimiento de la investigación la valoración por la comunidad científica de los hallazgos y desarrollos del conocimiento para la reproducción del sistema científico, y asimismo para la evaluación de la carrera investigadora (citaciones, sexenios).

A medida que pasan los años, las revistas mejor indexadas en Comunicación incrementan de forma progresiva la publicación de artículos vinculados con investigaciones pertenecientes a PI+D. Así se aprecia en el conjunto de la década estudiada (2007-2018). Obsérvese que después de un incremento muy importante desde 2011 hasta 2014, se estabiliza en 2014 hasta 2018, salvo el incremento de casi 5\% de las publicaciones en el año 2017. No disponemos de los datos suficientes para explicar esta desviación, cabe esperar que en secuencias longitudinales más largas la desviación desaparezca o se consolide la trayectoria ascendente.

\subsection{Análisis en las universidades y comunidades autónomas de las TD, los PI+D y artículos científicos}

En este apartado vamos a analizar los documentos seleccionados en función de las universidades y CC. AA., que nos servirán, en una segunda etapa de la investigación, para elaborar un mapa interactivo sobre el conjunto de la década 2007-2018. 
Por universidades, las TD analizadas se distribuyen del siguiente modo (cfr. tabla 5).

En la tabla 5 puede observarse que la Universidad Complutense de Madrid es la que lidera de manera incuestionable esta clasificación, pues una de cada cinco TD en Comunicación leídas en España en los últimos once años se han defendido en esta universidad; muy lejos, aunque en posiciones muy relevantes, segunda y tercera, están las universidades andaluzas de Sevilla y Málaga, respectivamente; la cuarta y quinta posición se corresponde con las universidades catalanas Autònoma de Barcelona y Pompeu Fabra. La segunda mitad de la tabla la encabeza, en sexta posición, la Rey Juan Carlos de Madrid, seguida por la primera universidad privada: la Ramon Llull; en octavo lugar aparece la Carlos III de Madrid, seguida por la Universidad del País Vasco (EHU); cerrando la tabla, en décima posición, aparecen dos universidades la de Santiago de Compostela y otra universidad privada de Madrid: la San Pablo CEU.

La desigualdad entre las universidades se manifiesta concretamente en primer lugar en el hecho de que sólo la UCM, con 586 TD, supera la mediana (293). Al comparar el número de tesis de cada universidad con la media $(53,6)$ hemos constatado que solo 14 de 48 universidades (el 29,1\%) está por encima de esa marca. Muchas de las universidades que están por debajo de la media han producido muy pocas, de hecho, hay 7 universidades que no han producido ninguna tesis doctoral (todas ellas de titularidad privada), 13 universidades con más de 20 y menos de 54 tesis, y 21 universidades con menos de 20 tesis en el periodo analizado.
Tabla 5. Universidades españolas con mayor rango por número de TD en la década

\begin{tabular}{|c|l|c|c|}
\hline Puesto & Universidad & No de tesis & Porcentaje \\
\hline 1 & UCM & 586 & 22,7 \\
\hline 2 & US & 198 & 7,7 \\
\hline 3 & UMA & 190 & 7,4 \\
\hline 4 & UAB & 180 & 7,0 \\
\hline 5 & UPF & 131 & 5,1 \\
\hline 6 & URJC & 93 & 3,6 \\
\hline 7 & URL & 91 & 3,5 \\
\hline 8 & UC3M & 89 & 3,5 \\
\hline 9 & UPV/EHU & 83 & 3,2 \\
\hline \multirow{2}{*}{10} & USC & 69 & 2,7 \\
\cline { 2 - 4 } & USPCEU & 69 & 2,7 \\
\hline
\end{tabular}

Tabla 6. Comunidades autónomas con mayor rango por número de TD en la década

\begin{tabular}{|c|l|c|c|}
\hline Puesto & CC. AA. & No de tesis & Porcentaje \\
\hline 1 & Madrid & 904 & 35,1 \\
\hline 2 & Catalunya & 479 & 18,6 \\
\hline 3 & Andalucía & 461 & 17,9 \\
\hline 4 & Valenciana (C.) & 210 & 8,1 \\
\hline 5 & Galicia & 114 & 4,4 \\
\hline 6 & Castilla y León & 101 & 3,9 \\
\hline 7 & País Vasco & 89 & 3,5 \\
\hline 8 & Navarra & 60 & 2,3 \\
\hline
\end{tabular}

Considerando las TD por CC. AA. (tabla 6) podemos observar que Madrid lidera la clasificación con poco más de 900 tesis. Con este dato se puede decir que una de cada tres TD que se han defendido en España en los últimos once años se ha llevado a cabo frente a un tribunal en una universidad de la Comunidad de Madrid.

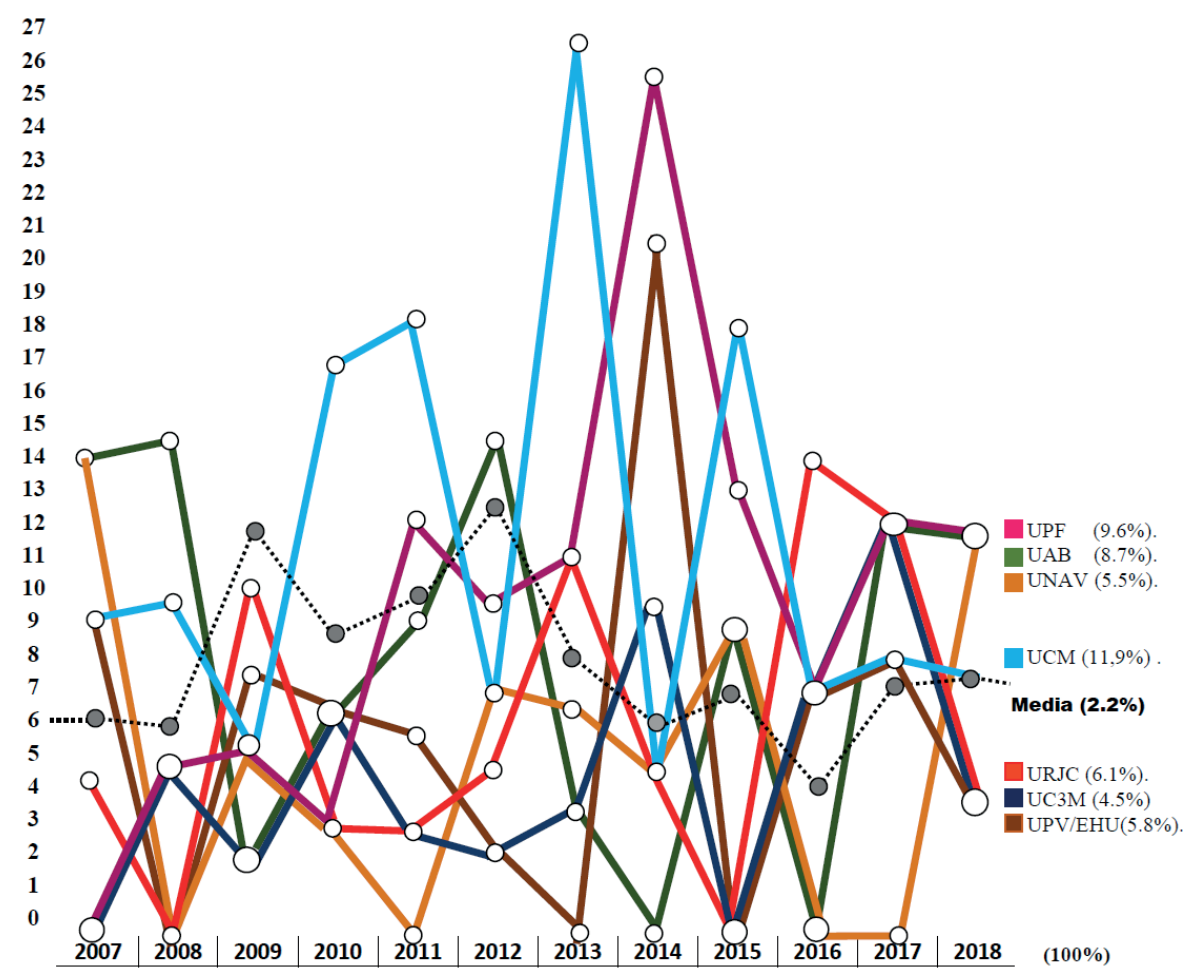

Gráfico 4. Universidades con mayor porcentaje de PI+D (2007-2018) 
Si trazamos la mediana (452 tesis) podemos observar que las universidades madrileñas, catalanas y andaluzas están por encima de ese punto. $\mathrm{Si}$ comparamos el número de tesis de cada comunidad autónoma con la media resultante $(171,8)$ podemos ver que las universidades valencianas se unen a las anteriores por encima de esa marca.

Un elemento a tener en cuenta para interpretar la clasificación por CC. AA. es el número de universidades por cada autonomía: Catalunya (11), Madrid (8); Valencia (6),

Andalucía (5), Castilla y León (4), Galicia (3), País Vasco, Murcia y Aragón (2); Baleares, Canarias, Castilla La Mancha, Extremadura, Navarra y La Rioja (1). Llama la atención que el número de universidades por CC. AA. no se corresponde con el rango de TD, y así, por ejemplo, Navarra con solo una universidad tiene 60 TD en tanto que Catalunya, con 11 universidades, apenas tiene la mitad de TD que la Comunidad de Madrid con 8 universidades.

Considerando los $\mathrm{PI}+\mathrm{D}$ por universidades y autonomías se aprecia por años un ascenso progresivo de las concesiones de proyectos I+D hasta 2012 (12,9\%) (cfr. gráfico 4).

Desde 2012 se produce un descenso paulatino (obsérvese la trayectoria descendente de la media en el gráfico 4, línea de puntos) que remonta en 2018 (8\%), de modo que el cómputo final queda prácticamente en los niveles del principio de la década estudiada, 2007 (7\%).

Por otra parte, cabe señalar que la evolución de las concesiones de financiación de PI+D de la década estudiada, presenta una configuración típica de dientes de sierra porque en el gráfico se puntúan los respectivos porcentajes que se corresponden a los años de cada convocatoria. Como los PI+D suelen otorgarse por una duración habitual de tres años, las universidades tienen que contar con suficiente número de equipos de investigación para cubrir las convocatorias en años sucesivos, lo que no ocurre si se carece de grupos de investigación suficientes compitiendo cada año. Concretamente las universidades que más se parecen a la trayectoria de la media por años son la UAB y la UPF, lo cual lleva a pensar que en ellas se está produciendo una mejor política científica en el apoyo estratégico a los grupos en las convocatorias.

Las CC. AA. que superan la media estatal respecto a la concesión de PI+D son: Madrid, Catalunya, Andalucía y Valencia (cfr. gráfico 5). Sólo si dividiéramos entre 17 autonomías (en vez de las 14 en las que hay representación con $\mathrm{PI}+\mathrm{D}$ ) entonces el País Vasco también entraría a formar parte del grupo de las autonomías que superan esa media en el conjunto del Estado. Vamos a considerar entonces en el grupo de cabeza al País Vasco, para poder incluirlo entre aquellas autonomías que vamos a evaluar año por año desde 2007 a 2018.

En conjunto, si analizamos en esta década las concesiones de $\mathrm{PI}+\mathrm{D}$, observamos que Catalunya resulta ser la primera autonomía (30,2\%) en número de concesiones y Madrid la segunda (27,3\%). Es decir, más de la mitad del peso de la investigación de los PI+D son acaparados por grupos de investigación de universidades pertenecientes a estas dos CC. AA. Quedan así relegadas, a un segundo nivel de asignación, Andalucía (9,3\%), Comunidad Valenciana $(7,7 \%)$ y finalmente, por debajo de la media (7,2\%) puede resaltarse la clase media-baja constituida por el País Vasco $(6,1 \%)$, Galicia (5,1\%), Navarra (4,8\%) y Castilla y León (3,5\%) (cfr. gráfico 6).

\subsection{Análisis de género en la autoría de las TD, de los PI+D y de los artículos científicos}

Atender la identidad de género en la investigación en comunicación supone que también en este campo existe una brecha de género como veremos en los análisis de los documentos de este trabajo. También es cumplir con lo preceptivo en las directrices emanadas del Parlamento Europeo en su programa Horizonte 2020, así como en los informes publicados por la Unidad de Mujer y Ciencia del Mineco.

La carrera universitaria de las mujeres sigue un itinerario en el que ellas representan una mayoría en el comienzo de los estudios, una casi equiparación con los hombres cuando se alcanza el grado de doctor y un declive muy notable en el acceso al nivel de cátedra (Caffarel et al., 2017).

Los datos sobre el género de los/as investigadores/as en las TD, como puede verse en la tabla 7 , constatan lo dicho anteriormente: poco más
Tabla 7. Género de la autoría de las TD

\begin{tabular}{|l|c|c|}
\cline { 2 - 3 } \multicolumn{1}{c|}{} & Frecuencia & Porcentaje \\
\hline Femenino & 1.360 & 52,8 \\
\hline Masculino & 1.217 & 47,2 \\
\hline Total & 2.577 & 100 \\
\hline
\end{tabular}




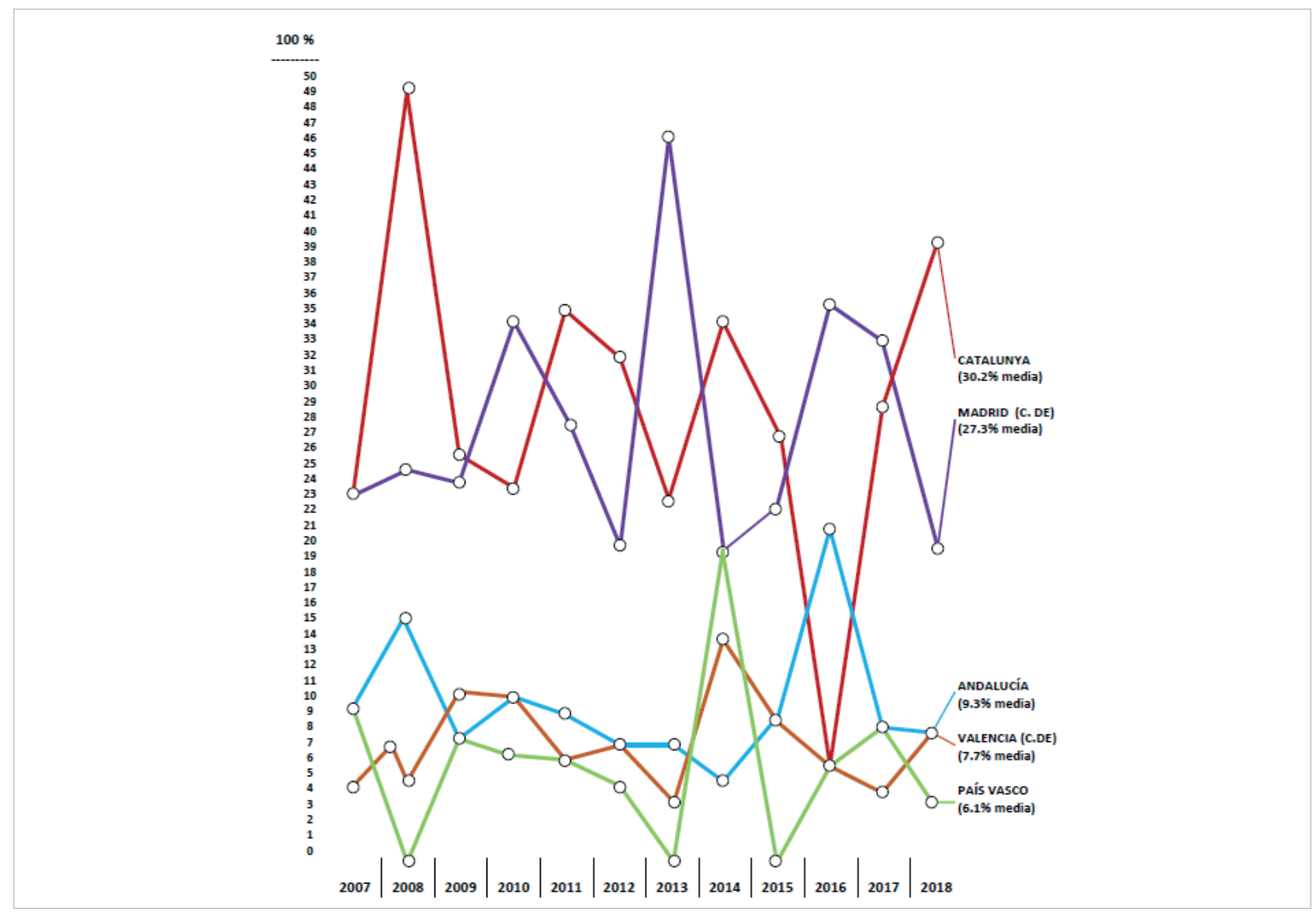

Gráfico 6. Autonomías que superan la media en la concesión de los PI+D de la década (2007-2018). Distribución en cada año.

de la mitad son mujeres $(52,8 \%)$ mientras que poco menos de la mitad son hombres $(47,2 \%)$.

Esta proporción genérica (poco más de la mitad de mujeres, poco menos de la mitad de hombres) no siempre se cumple si analizamos cada una de las universidades. En este sentido podemos decir que son muchas las universidades donde dos de cada tres tesis son defendidas por mujeres: UCJC, CEU-CH, UB, UDC, UDEUSTO, UGR, UDL, USAL, UVA, UVIGO, UNIZAR, UJI y URV. Solo dos universidades pueden decir que el $80 \%$ de sus doctorandas son mujeres: UEX y UAN.

Es importante mencionar que son muchas las universidades que mantienen la proporción genérica, pero en sentido inverso, es decir, poco más de la mitad de hombres, poco menos de la mitad mujeres. Son los casos de: UCAM, USPCEU, UA, ULL, UMU, USC, UMH, UPF, UPSA, URJC. Solo la UOC tiene el 70\% de doctorandos hombres durante el periodo analizado.

La dirección de $\mathrm{PI}+\mathrm{D}$ en la década (2007-2018) se muestra siempre a favor de los investigadores frente a las investigadoras (cfr. gráfico 7).

En este período la media de la desigualdad alcanza el 29.2\%. Las mujeres (línea roja) sólo se igualan a los hombres (línea azul) como IPs en los años 2008 y 2013, pero lo más frecuente es que esta diferencia ventajosa a favor de los varones sea persistente en el conjunto de la década y fluctúe entre el 20 y el $40 \%$. Nótese que la década comienza con una rela-

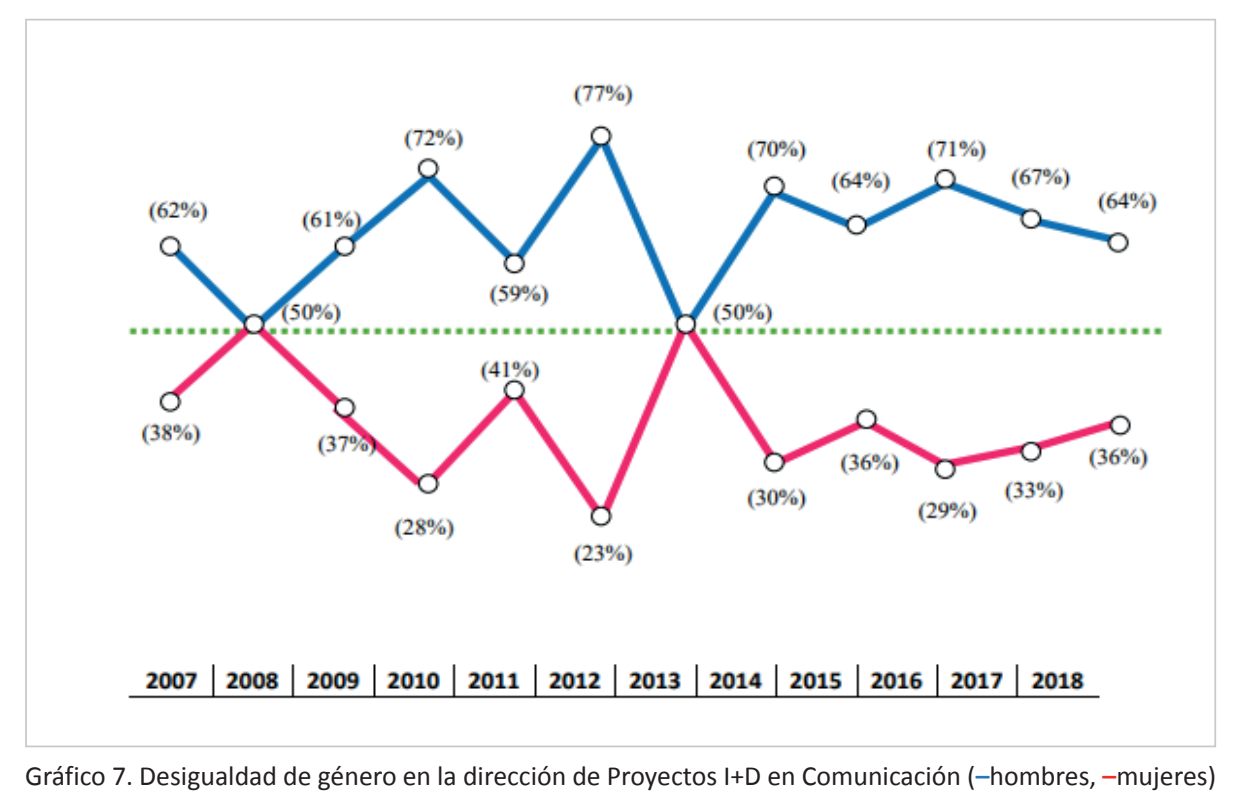


ción 62 vs. 38 y acaba con la correspondiente 64 vs. 36 (la media de la década es de 65 vs. 35) por lo que, en términos absolutos, no podemos hablar de ninguna mejora relevante en pro de la igualdad en la dirección de $\mathrm{PI}+\mathrm{D}$ en el área de los estudios sobre Comunicación. No obstante, desde 2016 la tendencia que se aprecia es la de un progresivo mejor reparto de género en la dirección de este tipo de proyectos.

Del análisis del género en la autoría de los artículos científicos se desprenden los datos representados en el gráfico 8.

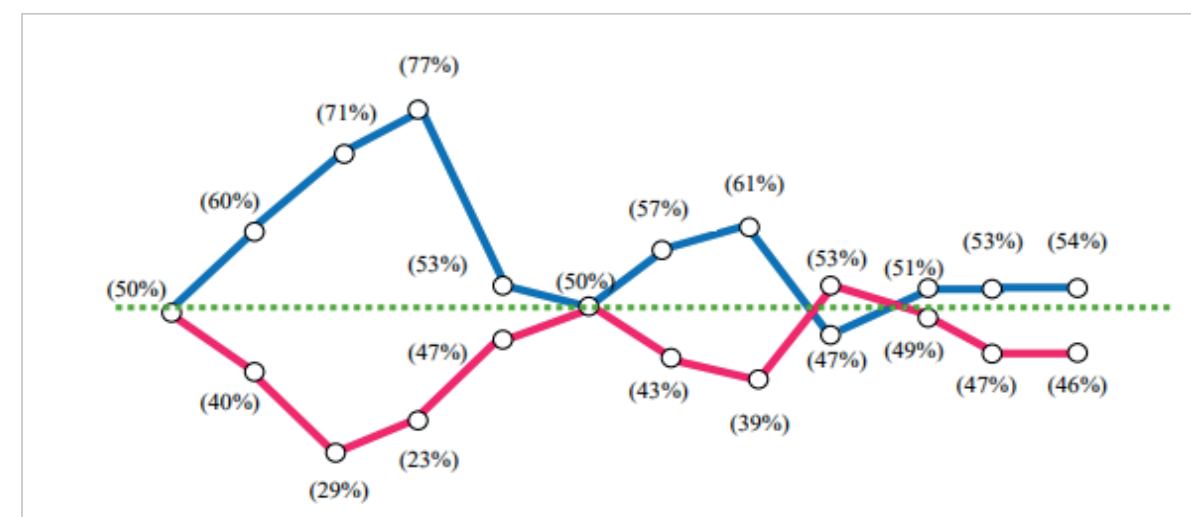

\begin{tabular}{l|l|l|l|l|l|l|l|l|l|l|l}
2007 & 2008 & 2009 & 2010 & 2011 & 2012 & 2013 & 2014 & 2015 & 2016 & 2017 & 2018 \\
\hline
\end{tabular}

Gráfico 8. Desigualdad de género en la publicación de artículos (primera autoría) en las revistas mejor indexadas de Comunicación (-hombres, -mujeres)

En lo que se refiere a la autoría de artículos en las revistas españolas mejor indexadas en Comunicación en la década (2007-2018), el gráfico muestra la máxima desigualdad de género entre los años 2009 y 2010 y entre 2013 y 2014, siempre a favor de los autores frente a las autoras, siendo la media de esta desigualdad el 8,4\%. Hay excepciones, por ejemplo, en el año 2015 se produce una inversión de tal primacía en $6 \%$ a favor de las autoras. Las mujeres además consiguen igualarse a los hombres a este respecto en los años 2007 y 2012.

Hay que señalar que entre las revistas españolas mejor indexadas Comunicar (52,9\% vs. 47,1\%), Comunicación y sociedad (56,2\% vs. 43,8\%) y Trípodos (58,8\% vs. 41,2\%) presentan una proporción de primeras firmas superior en las autoras que en los autores de los artículos.

\subsection{Análisis comparativo del volumen de documentos analizados a partir de las variables de tiempo, auto- nomías y universidades y género}

Para tener una visión global de la investigación en Comunicación en España durante el periodo de 2007 a 2018 vamos a comparar y comentar el volumen total de cada uno de los documentos analizados (TD, PI+D, artículos) y añadiendo además sumamos el volumen del repositorio de comunicaciones a congresos de la $A E-I C$ por entender que es el conjunto total de datos el que proporciona la instantánea de lo que producen los investigadores en Comunicación en la universidad española con Facultades para titulaciones de grado y postgrado en Comunicación..

Tabla 8. Volumen total de documentos por bienios

\begin{tabular}{|c|c|c|c|c|c|c|c|c|c|c|}
\hline & Artículos & $\%$ & Coms. & $\%$ & $\mathrm{PI}+\mathrm{D}$ & $\%$ & TD & $\%$ & Total & $\%$ \\
\hline $2007-08$ & 7 & 1,7 & 191 & 22,4 & 41 & 13,0 & 132 & 5,1 & 371 & 8,8 \\
\hline 2009-10 & 20 & 4,9 & 107 & 12,5 & 67 & 21,4 & 282 & 11,0 & 476 & 11,4 \\
\hline 2011-12 & 51 & 12,3 & 182 & 21,4 & 72 & 22,8 & 392 & 15,2 & 697 & 16,7 \\
\hline 2013-14 & 100 & 24,2 & 131 & 15,4 & 47 & 15,0 & 351 & 13,6 & 629 & 15,1 \\
\hline 2015-16 & 108 & 26,1 & 135 & 15,8 & 39 & 12,3 & 945 & 36,6 & 1227 & 30,7 \\
\hline 2017-18 & 127 & 30,8 & 107 & 12,5 & 49 & 15,5 & 475 & 18,5 & 758 & 18,2 \\
\hline Total & 413 & 100 & 853 & 100 & 315 & 100 & 2.577 & 100 & 4.158 & 100 \\
\hline
\end{tabular}

Como se observa en la tabla 8 hemos optado por la secuencia temporal bienal, como hemos explicado en la metodología, porque es la única que nos permite la comparación equidistante; no olvidemos que los congresos de la $A E-I C$ se llevan a cabo cada dos años. Del volumen de datos cabe deducir que las comunicaciones y las TD (quitando el fenómeno ya explicado del año 2016 que dispara su producción) se mantienen más o menos estables en el tiempo; no ocurre de la misma manera con los artículos publicados en las revistas indexadas donde van creciendo paulatinamente en tiempo. Si tenemos en cuenta que los datos del bienio 2007-08 muestran una proporción de casi seis PI+D por artículo, en el bienio 2017-18, justo una década después, se invierten los datos y por cada PI+D se publican 2,5 artículos en revistas indexadas, por tanto, cabe entender pues que, para la transferencia de los resultados de los proyectos nacionales, los investigadores eligen más el documento artículo.

Los datos reflejados por comunidades autónomas y universidades tienen un volumen tan grande que parece pertinente reflejar las comunidades de Madrid y Catalunya por ser las que tienen mayor número de universidades (cfr. tablas 9 y 10). 
Tabla 9. Volumen total de documentos en las universidades catalanas

\begin{tabular}{|c|c|c|c|c|c|c|c|c|c|c|c|}
\hline \multirow{14}{*}{ Catalunya } & Universidad & $\begin{array}{l}\text { Artícu- } \\
\text { los }\end{array}$ & $\%$ & Coms. & $\%$ & $\mathrm{PI}+\mathrm{D}$ & $\%$ & TD & $\%$ & Total & $\%$ \\
\hline & Abat Oliba CEU & 0 & 0 & 0 & 0 & 0 & 0 & 10 & 2,2 & 10 & 1,2 \\
\hline & Autònoma de Barcelona & 24 & 25,0 & 68 & 40,7 & 31 & 31 & 180 & 37,7 & 303 & 36,1 \\
\hline & Barcelona & 11 & 11,5 & 0 & 0 & 12 & 12 & 0 & 0 & 23 & 2,7 \\
\hline & Girona & 1 & 1,0 & 3 & 1,8 & 1 & 1 & 17 & 3,5 & 22 & 2,6 \\
\hline & Lleida & 2 & 2,0 & 1 & 0,6 & 2 & 2 & 5 & 1,0 & 10 & 1,2 \\
\hline & Vic & 1 & 1,0 & 8 & 4,8 & 1 & 1 & 9 & 1,8 & 19 & 2,3 \\
\hline & $\begin{array}{l}\text { Internacional de Cata- } \\
\text { lunya }\end{array}$ & 2 & 2,0 & 2 & 1,2 & 0 & 0 & 4 & 0,8 & 9 & 1,1 \\
\hline & Oberta de Catalunya & 7 & 7,3 & 19 & 11,3 & 5 & 5 & 7 & 1,5 & 38 & 4,5 \\
\hline & Politècnica de Catalunya & 0 & 0 & 2 & 1,2 & 0 & 0 & 0 & 0 & 2 & 0,2 \\
\hline & Pompeu Fabra & 35 & 36,5 & 30 & 18 & 30 & 30 & 131 & 27,5 & 226 & 26,9 \\
\hline & Ramon Llull & 8 & 8,4 & 16 & 9,6 & 8 & 8 & 91 & 19 & 123 & 14,6 \\
\hline & Rovira i Virgili & 5 & 5,3 & 18 & 10,8 & 8 & 8 & 24 & 5 & 55 & 6,5 \\
\hline & Total & 96 & 100 & 167 & 100 & 98 & 100 & 478 & 100 & 840 & 100 \\
\hline
\end{tabular}

Tabla 10. Volumen total de documentos en las universidades madrileñas

\begin{tabular}{|c|c|c|c|c|c|c|c|c|c|c|c|}
\hline \multirow{14}{*}{ Madrid } & Universidad & $\begin{array}{c}\text { Artícu- } \\
\text { los }\end{array}$ & $\%$ & Coms. & $\%$ & $\mathrm{PI}+\mathrm{D}$ & $\%$ & TD & $\%$ & Total & $\%$ \\
\hline & IE University & 0 & 0 & 5 & 2,4 & 2 & 2,4 & 14 & 1,5 & 21 & 1,6 \\
\hline & Alcalá de Henares & 1 & 1,1 & 0 & 0 & 1 & 1,2 & 0 & 0 & 2 & 0,2 \\
\hline & Antonio de Nebrija & 0 & 0 & 4 & 2 & 0 & 0 & 10 & 1,1 & 14 & 1,0 \\
\hline & Autónoma de Madrid & 0 & 0 & 0 & 0 & 2 & 2,4 & 0 & 0 & 2 & 0,2 \\
\hline & Camilo José Cela & 1 & 1,1 & 4 & 2 & 0 & 0 & 23 & 2,6 & 28 & 2,1 \\
\hline & Carlos III de Madrid & 15 & 16,4 & 37 & 17,8 & 14 & 16,8 & 89 & 9,8 & 155 & 12,0 \\
\hline & CEU San Pablo & 4 & 4,4 & 18 & 8,6 & 6 & 7,2 & 69 & 7,6 & 97 & 7,6 \\
\hline & Complutense de Madrid & 35 & 38,5 & 73 & 35,0 & 37 & 44,5 & 586 & 64,8 & 731 & 56,8 \\
\hline & Europea de Madrid & 0 & 0 & 3 & 1,4 & 0 & 0 & 20 & 2,3 & 23 & 1,7 \\
\hline & Nacional a Distancia & 4 & 4,4 & 0 & 0 & 0 & 0 & 0 & 0 & 4 & 0,5 \\
\hline & Politécnica de Madrid & 3 & 3,3 & 0 & 0 & 1 & 1,2 & 0 & 0 & 4 & 0,5 \\
\hline & Rey Juan Carlos & 28 & 30,8 & 61 & 29,2 & 21 & 25,3 & 93 & 10,3 & 203 & 15,8 \\
\hline & Total & 91 & 100 & 209 & 100 & 83 & 100 & 904 & 100 & 1287 & 100 \\
\hline
\end{tabular}

Al analizar los datos lo primero que cabe observar es que aparecen universidades que no tienen grados o postgrados en Comunicación, son las Universidades de Alcala, Autónoma de Madrid y la Politécnica de Madrid (cfr. tabla 10). En estas tres universidades encontramos artículos científicos ligados a PI+D, lo que lleva a pensar en la pluridisciplinariedad para abordar los fenómenos comunicativos objetos de investigaciones nacionales. El segundo dato que cabe destacar es el diferente comportamiento entre las universidades públicas y privadas; las universidades privadas, a excepción de la Universidad CEU San Pablo en la Comunidad de Madrid y la Universitat Oberta de Catalunya, no aportan artículos científicos derivados de PI+D ni PI+D, tan sólo y con un número no significativo, aparecen por las TD defendidas y por comunicaciones presentadas a los congresos de la $A E-I C$ que no tienen por qué estar ligados a $\mathrm{PI}+\mathrm{D}$ ya que hemos trabajado con el total de las ponencias presentadas.

Por último, hemos querido presentar una radiográfica, por la variable género, del conjunto de documentos que caracterizan la investigación en comunicación en España ligada a las universidades con grados o postgrados en comunicación (tabla 11).

Del análisis cabe deducir que en aquellos documentos que son más relevantes para la carrera investigadora, a saber, $\mathrm{PI}+\mathrm{D}$ y su transferencia en artículos publicados en revistas indexadas, los hombres siguen predominando con un porcentaje de $65,1 \%$ para PI+D, y de 54,2\% en artículos científicos, en tanto que para las TD y las comunicaciones a congresos la proporción se invierte: un 52,8\% para las TD y un 51,3\% de comunicaciones a favor de las mujeres. Cabe pensar que, como hemos visto en el análisis de las secuencias temporales, se está produciendo una ligera mejora en la penetración de la mujer a la investigación de alto rendimiento ( $\mathrm{PI}+\mathrm{D}$ y artículos en revistas indexadas), pero es un incremento lento que no ha llegado ni tan siquiera a la paridad y por tanto reflejando el techo de cristal que se da no sólo en la investigación sino en otros ámbitos de la vida social. 
Tabla 11. Volumen total de documentos por género

\begin{tabular}{|c|c|c|c|c|c|c|c|c|c|c|}
\hline & \multicolumn{2}{|c|}{ Artículos } & \multicolumn{2}{|c|}{ Comunicaciones } & \multicolumn{2}{|c|}{$\mathrm{PI}+\mathrm{D}$} & \multicolumn{2}{|c|}{ TD } & \multicolumn{2}{|c|}{ Total } \\
\hline \multirow{3}{*}{ Femenino } & \multicolumn{2}{|c|}{189} & \multicolumn{2}{|c|}{438} & \multicolumn{2}{|c|}{110} & \multicolumn{2}{|c|}{1.360} & \multicolumn{2}{|c|}{2.097} \\
\hline & $9,0 \%$ & & $20,9 \%$ & & $5,2 \%$ & & $64,9 \%$ & & $100 \%$ & \\
\hline & & $45,8 \%$ & & $51,3 \%$ & & $34,9 \%$ & & $52,8 \%$ & & $50,4 \%$ \\
\hline \multirow{3}{*}{ Masculino } & \multicolumn{2}{|c|}{224} & \multicolumn{2}{|c|}{415} & \multicolumn{2}{|c|}{205} & \multicolumn{2}{|c|}{1217} & \multicolumn{2}{|c|}{2.061} \\
\hline & $10,9 \%$ & & $20,1 \%$ & & $9,9 \%$ & & $59,0 \%$ & & $100 \%$ & \\
\hline & & $54,2 \%$ & & $48,7 \%$ & & $65,1 \%$ & & $47,2 \%$ & & $49,6 \%$ \\
\hline \multirow{3}{*}{ Total } & \multicolumn{2}{|c|}{413} & \multicolumn{2}{|c|}{853} & \multicolumn{2}{|c|}{315} & \multicolumn{2}{|c|}{2.577} & \multicolumn{2}{|c|}{4.158} \\
\hline & $9,9 \%$ & & $20,5 \%$ & & $7,6 \%$ & & $62,0 \%$ & & $100 \%$ & \\
\hline & & $100 \%$ & & $100 \%$ & & $100 \%$ & & $100 \%$ & & $100 \%$ \\
\hline
\end{tabular}

\section{Discusión y conclusiones}

En esta exposición hemos comenzado resaltando la importancia de conocer la evolución de la productividad investigadora sobre las prácticas sociales de la comunicación llevada a cabo por investigadores adscritos a universidades españolas con titulaciones de grados y postgrados del campo de la Comunicación: Periodismo, Publicidad y RR. Públicas, Comunicación Audiovisual y Documentación. Para ello hemos señalado como objeto de estudio la evolución temporal de los perfiles cartográficos de autonomías y universidades y de identidad de género atendiendo a la autoría masculina vs. femenina en los tres tipos de producciones intelectuales de mayor prestigio académico universitario: tesis doctorales (TD), proyectos de investigación I+D+I (PI+D) de convocatorias estatales, y artículos publicados en revistas científicas de impacto. Todo ello remitido al plazo temporal de una década: 2007-2018.

Hemos señalado en este sentido que la relevancia de estudiar esta productividad deriva de considerar cuáles son los rangos del reconocimiento social de la actividad investigadora destinada a la transferencia social de conocimientos, de la cual depende la legitimación de las aplicaciones de la investigación realizada, la reproducción del sistema científico de investigación y la cooptación académica de los mejores investigadores. Con este objetivo se ha revisado cuál ha sido el contexto de este enfoque de metainvestigación en el campo de los estudios de las prácticas sociales de comunicación.

Para abordar la construcción de los repositorios de TD, PI+D y artículos científicos se ha expuesto la metodología empleada para la elaboración de datos a partir de fuentes secundarias y se han presentado los datos más relevantes, primero de la evolución temporal por años de dichos documentos, segundo, de los perfiles de su identificación por universidades y comunidades autónomas; y tercero, finalmente, de la identidad de género de su autoría.

Presentados y comentados los datos más relevantes de la comparación entre TD, PI+D, artículos por evolución temporal, universidades, comunidades autónomas y género, pueden someterse a discusión las siguientes conclusiones:

1) Por su evolución temporal, en la década 2007-2018, las TD se acumulan en torno al $50 \%$ en el año 2016, como consecuencia de la imposición de plazos de cinco años para culminar antes de finalizar 2016, el proceso de investigación previo a la defensa de una tesis doctoral. Por ello, los doctorandos se vieron obligados por el cambio de normativa a presentar antes de finalizar 2016 la defensa de las TD iniciadas antes de 2011. El análisis que posteriormente nos emplazamos a realizar tomará en cuenta la cuestión de si esa imposición de plazos rígidos para culminar los procesos de investigación lleva a reducir su calidad y si esto se refleja en las calificaciones obtenidas durante el pico de 2016.

Igualmente, por su evolución temporal, los PI+D nos han conducido a cuestionarnos el decurso de las políticas científicas de financiación de la investigación, pues si tras la crisis de 2008 se mantiene el incremento de Pl+D, en cambio a partir de 2012 se precipitan hasta hundirse en porcentajes similares a los años anteriores a la década precedente.

También por su evolución temporal, los datos sobre los artículos publicados en revistas científicas de impacto nos han permitido descubrir una curva ascendente en su presencia, teniendo en cuenta que se han considerado sólo aquellos artículos vinculados a PI+D, destacando su disparado incremento seguramente como consecuencia de la competencia exigida por las agencias de evaluación en el curso de la década.

2) Por la localización de universidades y CC. AA. a que están adscritos los autores de TD e investigadores de PI+D, así como autores de artículos en revistas científicas y comunicaciones en congresos, se ha resaltado que son las universidades de las CC. AA. de Madrid, Cataluña y Andalucía las que concentran casi la totalidad de las TD, hasta el punto de que sólo la Comunidad de Madrid suma el doble de las tesis defendidas en Cataluña, por ejemplo, o en Andalucía. Esta desigual distribución es fundamentalmente derivada del número de universidades que integran cada una de estas Comunidades, pero de forma especial por efecto de la diversidad en sus políticas científicas, ya que no se corresponde un mayor número de universidades por comunidad autónoma con un mayor número de tesis, pues Cataluña, por ejemplo, produjo la mitad de TD que Madrid con el mismo número de universidades. Finalmente, la aportación de TD por univer- 
sidades y CC. AA. depende sobre todo de la antigüedad y envergadura de la población de alumnos de postgrado y de la implementación de programas de tercer ciclo. Destacando la UAB y la UPF en Cataluña, la UCM en Madrid y la UMA y la US en Andalucía.

Los PI+D en relación con las universidades y sus CC. AA. muestran también efectos de las políticas científicas que acompañan a las convocatorias estatales. Así, resalta que contemplando la curva del número de PI+D localizados por el año de su convocatoria, hay universidades cuya curva presenta dientes de sierra como efecto de que carecen de suficiente número de equipos de investigación capaces de cubrir año a año todas las convocatorias. Aquellas universidades cuya trayectoria en el número de proyectos se aleja más del perfil de la curva representada por la media de proyectos anuales son supuestamente las que más carecen de una dirección estratégica en la gestión de su política científica.

3) Considerando la identidad de género de investigadores y firmantes de artículos y de comunicaciones, no se observa a lo largo de la década una disminución indicativa de la brecha de género. Ahora bien, si analizamos separadamente los documentos, observamos que es en la autoría de las TD donde la diferencia es menor, incluso la media de mujeres doctorandas resulta superior en muchas universidades, como la UCJC, CEU-CH, UB, UDC, UDEUSTO, UGR, UDL, USAL, UVA, UVIGO, UNIZAR, UJI y URV, alcanzando el $80 \%$ de sus doctorandas en la UEX y en la UAN. Estos datos no hacen más que corroborar que es en los estudios de doctorado donde se iguala el número de doctorandas y doctorandos, como señala el libro blanco sobre la situación de las mujeres en la ciencia española (Sánchez-de-Madariaga, 2018) al evaluar la presencia de mujeres científicas en el panorama universitario nacional. Sin embargo, cuando hablamos de PI+D, artículos en revistas de impacto y comunicaciones de congresos, encontramos otros datos relevantes. A saber, la escala de la desigualdad va pareja al prestigio que la comunidad científica otorga a los documentos analizados y a la categoría profesional del investigador o investigadora. Así, en la dirección de los PI+D resulta muy desigual, muy desventajosa para las IP, mientras que en la autoría de los artículos la distancia de género es más moderada, aun a pesar de la mayor presencia de los autores frente a las autoras; y finalmente, en la autoría de las comunicaciones a congresos, es donde la diferencia es casi inexistente. En definitiva, la presencia de la mujer en el conjunto de los documentos analizados incita a pensar que no se siguen las recomendaciones que emite la Unión Europea en su Programa Horizonte 2020 en cuyo objetivo primero es lograr el equilibrio de género en los equipos de investigación a todos los niveles.

Descendiendo al detalle de los datos, en los PI+D, en el conjunto de la década, la diferencia a favor de los varones fluctúa entre el 20 y el $40 \%$. En el caso de las revistas de impacto, porque contradice la tendencia general, llama la atención que tres de ellas, ciertamente muy valoradas, presenten una proporción de primeras firmas superior en las autoras que en los autores: Comunicar (52,9\% vs. 47,1\%), Comunicación y sociedad (56,2\% vs. 43,8\%) y Trípodos (58,8\% vs. 41,2\%).

En último lugar hemos presentado el conjunto de datos que hemos obtenido en MapCom del vaciado de TD, $\mathrm{PI}+\mathrm{D}$, artículos científicos y, en este caso también, las ponencias presentadas a los congresos de la $A E-I C$, para mostrar el volumen de la producción científica en comunicación en España desde 2007 a 2018 que supone un universo de 4.158 documentos. De esta radiografía cabe extraer las siguientes conclusiones: Hay un crecimiento exponencial en la secuencia analizada de artículos publicados en revistas científicas de impacto; por el contrario las comunicaciones a los congresos de la $A E-I C$ experimentan, durante la década, una ilustrativa tendencia a decrecer, precisamente en concordancia con la progresiva minusvaloración de las comunicaciones a congresos en el reconocimiento de la actividad investigadora, por parte de las agencias de evaluación ( $\mathrm{vg}$. la CNEAl); merece la pena destacar que la declinante participación de investigadores de distintas universidades aportando comunicaciones en los congresos de la $A E$ - IC ofrece una visión decadente del papel de estas convocatorias, poniendo en cuestión la viabilidad de su sostenibilidad, pues si en otros campos, como por ejemplo en Medicina o en Física, la previa presentación y discusión de resultados de una investigación en congresos supone un aval para su publicación posterior en revistas de prestigio científico, en nuestro campo, incomprensiblemente, las agencias evaluadoras minusvaloran la publicación en actas de congresos, induciendo a los autores a disminuir su participación en estos congresos, y a retirar de la publicación de actas los textos de los avances comunicados de su investigación.

Las universidades públicas son las garantes de la investigación de alto nivel ya que aglutinan un porcentaje muy significativo de $\mathrm{PI}+\mathrm{D}$ nacionales y de publicaciones en revistas científicas de impacto, en tanto que las privadas aparecen con un porcentaje mayor, en ocasiones único, de TD y comunicaciones a congresos, salvo la excepción muy notoria de las Universidades de Navarra, Oberta de Catalunya y CEU San Pablo que obtienen datos importantes en PI+D y artículos científicos.

Respecto al género, teniendo como referente el volumen total de datos presentados en las distintas categorías de documentos, los hombres sobresalen, frente a las mujeres, en el liderazgo de PI+D y de publicaciones en revistas de impacto; la tendencia se invierte, a favor de las mujeres, respecto al volumen total de TD y comunicaciones a congresos, aunque viendo el análisis longitudinal la mujer va escalando poco a poco puestos en la visibilidad de su labor investigadora.

Finalmente, a falta de continuar verificando datos, notamos una desigualdad, no solo de género, sino también de políticas científicas en las universidades y CC. AA. La trayectoria de ambas, respecto al apoyo a la investigación, debería ser mucho más homogénea entre ellas y sin embargo se observan muchas desigualdades. No obstante, considerando el análisis longitudinal, observamos tendencias en la investigación universitaria en Comunicación en España que pueden suponer, como hipótesis para futuros proyectos, algún cambio positivo en el horizonte. 


\section{Referencias}

Arcila-Calderón, Carlos; Piñuel-Raigada, José-Luís; Calderín-Cruz, Mabel (2013). "The e-research on media \& communications: Attitudes, tools and practices in Latin America researchers". Comunicar, v. 40, pp. 111-118.

https://doi.org/10.3916/C40-2013-03-01

Barranquero-Carretero, Alejandro; Limón-Serrano, Nieves (2017). “Objetos y métodos dominantes en comunicación para el desarrollo y el cambio social en las tesis y proyectos de investigación en España (2007-2013)". Revista latina de comunicación social, n. 72, pp. 1-25.

https://doi.org/10.4185/RLCS-2017-1151

Blázquez-Ochando, Manuel (2015). Tesis doctorales en las universidades españolas durante el periodo 1977-2014. http://mblazquez.es/tesis-doctorales-en-las-universidades-espanolas-durante-el-periodo-1977-2014

Cáceres-Zapatero, María-Dolores; Caffarel-Serra, Carmen (1993). “La investigación en comunicación en España. Un balance cualitativo". Telos, v. 32, pp. 109-124.

Caffarel-Serra, Carmen; Domínguez, Milagros; Romano, Vicente (1989). "El estado de la investigación en comunicación en España (1978-1987)". Cuadernos de investigación en comunicación, n. 3, pp. 45-57.

Caffarel-Serra, Carmen; Gaitán-Moya, Juan-Antonio; Lozano-Ascencio, Carlos; Piñuel-Raigada, José-Luis (2018a). Tendencias metodológicas en la investigación académica sobre comunicación. Salamanca: Comunicación Social, 301 pp. ISBN: 9788415544692

Caffarel-Serra, Carmen; Ortega, Félix; Gaitán-Moya, Juan-Antonio (2018b): “La investigación en comunicación en España: debilidades, amenazas, fortalezas y oportunidades”. Comunicar, v. XXVI, n. 56, pp. 61-70.

https://doi.org/10.3916/C56-2018-06

Caffarel-Serra, Carmen; Ortega, Félix; Gaitán-Moya, Juan-Antonio (2017). “Investigación en comunicación en la universidad española en el periodo 2007-2014". El profesional de la información, v. 26, n. 2, pp. 218-227.

https://doi.org/10.3145/epi.2017.mar.08

Caffarel-Serra, Carmen; Piñuel-Raigada, José-Luis; Lozano-Ascencio, Carlos; Gaitán-Moya, Juan-Antonio (2019a). "Base general de datos MapCom sobre proyectos I+D y tesis doctorales realizados en España de 2007 a 2013 ". e-cienciaDatos, V1.

https://doi.org/10.21950/ZQRFFA

Caffarel-Serra, Carmen; Piñuel-Raigada, José-Luis; Lozano-Ascencio, Carlos; Gaitán-Moya, Juan-Antonio (2019b). "Base de datos encuesta MapCom". e-cienciaDatos, V1.

https://doi.org/10.21950/YRHVOW

Castillo-Esparcia, Antonio; Carretón-Ballester, María-Carmen (2010). “Investigación en comunicación. Estudio bibliométrico de las revistas de comunicación en España”. Comunicación y sociedad, v. 23, n. 2, pp. $289-327$.

https://hdl.handle.net/10171/27872

Castillo-Esparcia, Antonio; Xifra, Jordi (2006). "Investigación bibliométrica de las tesis doctorales españolas sobre relaciones públicas (1965-2005)". Anàlisi, v. 34, pp. 141-161.

https://www.raco.cat/index.php/Analisi/article/view/55449

De-Filippo, Daniela (2013). "La producción científica española en comunicación en WoS. Las revistas indexadas en SSCI (2007-2012). Comunicar, v. XXI, n. 41, pp. 25-34.

https://doi.org/10.3916/C41-2013-02

Delgado-López-Cózar, Emilio; Ayllón, Juan-Manuel, Ruiz-Pérez, Rafael (2013). Índice h de las revistas científicas españolas según Google Scholar Metrics (2007-2011) (2a ed.). EC3 Informes, 3, 9 de abril.

https://cutt.ly/Oruqklo

Delgado-López-Cózar, Emilio; Martín-Martín, Alberto (2018). Índice h de las revistas científicas españolas según Google Scholar Metrics (2013-2017) (2-a ed.). Universidad de Granada, 18 diciembre.

https://digibug.ugr.es/handle/10481/54306

Delgado-López-Cózar, Emilio; Torres-Salinas, Daniel; Jiménez-Contreras, Evaristo; Ruiz-Pérez, Rafael (2006). “Análisis bibliométrico y de redes sociales aplicado a las tesis bibliométricas defendidas en España (1976-2002): temas, escuelas científicas y redes académicas". Revista española de documentación científica, v. 29, n. 4, pp. 493- 524.

https://doi.org/10.3989/redc.2006.v29.i4.306

Díaz-Nosty, Bernardo; De-Frutos-García, Ruth (coords.) (2017). Tendencias de la investigación universitaria española en comunicación. Ed. Thomson Reuters, ISBN: 9788491357193 
Férnandez-Quijada, David; Masip-Masip, Pere (2013). “Tres décadas de investigación española en comunicación: hacia la mayoría de edad". Comunicar, v. 41, pp. 15-24.

https://doi.org/10.3916/C41-2013-01

Fuentes-Navarro, Raúl (1994). "La investigación de la comunicación ¿̇hacia la postdisciplinariedad en ciencias sociales?”. En Lameiras y Galindo (eds.) Medios y mediaciones, Guadalajara. México: El Colegio de Michoacán/Iteso. ISBN: 9686101411

Fuentes-Pujol, Eulàlia; Arguimbau-Vivó, Llorenç (2010). “Las tesis doctorales en España (1997-2008): análisis, estadísticas y repositorios cooperativos". Revista española de documentación científica, v. 33, n. 1, pp. 63-89.

https://doi.org/10.3989/redc.2010.1.711

Gaitán-Moya, Juan-Antonio (2014). "Investigación universitaria e investigación de mercado en comunicación: comparación y contraste en España”. IV Congreso internacional de la AE-IC: Espacios de comunicación, Bilbao, 21-24 de enero, pp. 1779-1790.

https://drive.google.com/file/d/1fsJ4ApS8vclvIGq7svvSNa1k2aef76Zn/view

Gaitán-Moya, Juan-Antonio; Caffarel-Serra, Carmen; Lozano-Ascencio, Carlos; Piñuel-Raigada, José-Luis (2018). “Condiciones y rendimientos de la investigación en comunicación: la visión de los académicos". CIC. Cuadernos de información y comunicación, v. 23, pp. 105-123.

https://doi.org/10.5209/CIYC.60910

Gaitán-Moya, Juan-Antonio; Lozano-Ascencio, Carlos; Piñuel, José-Luis (2016). "Prospectiva de la investigación sobre TV a partir de los proyectos I+D y tesis doctorales de 2007 a 2013 en las facultades españolas de comunicación". Revista Asociación Española de Comunicación, v. 6, n. 3, pp. 51-59. http://www.revistaeic.eu/index.php/raeic/article/view/66

Giménez-Toledo, Elea; Jiménez-Contreras, Evaristo (2013). “Los agujeros negros de la comunicación: Comunicación científica y metainvestigación". Comunicar, v. XXI, n. 41.

https://doi.org/10.3916/C41-2013-a2

Horizonte 2020. Igualdad de género en la investigación y la innovación.

http://www.eshorizonte2020.es/mas-europa/ciencia-con-y-para-la-sociedad/igualdad-de-genero-en-la-investigaciony-la-innovacion

Jones, Daniel E. (1994). Investigació sobre comunicació a l'Espanya dels noranta. Centre d'Investigació de la Comunicació i Universitat Autònoma de Barcelona. Cultura y comunicación social: América Latina y Europa Ibérica. Barcelona: Generalitat de Catalunya.

Jones, Daniel E. (1998). “Investigación sobre comunicación en España. Evolución y perspectivas”. Zer, n. 5, pp. 13-51. https://www.ehu.eus/ojs/index.php/Zer/article/view/17359

López-Escobar, Esteban; Martín-Algarra, Manuel (2016). "Communication teaching and research in Spain". Publizistik, v. 62, pp. 83-105.

https://doi.org/10.1007/s11616-016-0306-4

Lozano-Ascencio, Carlos; Gaitán-Moya, Juan-Antonio (2011). “Dedicación a la investigación y a la docencia universitaria en comunicación: compatibilidad y perfiles en España, Europa y América Latina”. En: Piñuel, José-Luis; Lozano, Carlos; García, Alberto (eds.). Investigar la comunicación en España (pp. 587-595). Facultad de CC. de la Comunicación. Fuenlabrada: Universidad Rey Juan Carlos.

Lozano-Ascencio, Carlos; Gaitán-Moya, Juan Antonio (2016). "Vicisitudes de la investigación en comunicación en España en el sexenio 2009-2015". Anuario electrónico de estudios en comunicación social "Disertaciones", v. 9, n. 2, pp. 139-162.

https://doi.org/10.12804/ disertaciones

Martín-Algarra, Manuel; Serrano-Puche, Javier; Rebolledo, Marta (2018). "La mujer en la investigación en comunicación en España: un análisis de la producción científica (2007-2013)”. adComunica, n. 15, pp. 65-87.

https://doi.org/10.6035/2174-0992.2018.15.5

Martínez-Nicolás, Manuel (2009). "La investigación sobre comunicación en España. Evolución histórica y retos actuales”. Revista latina de comunicación social, v. 64, pp.1-14. https://doi.org/10.4185/RLCS-64-2009-800-01-14

Martínez-Nicolás, Manuel; Saperas, Enric (2011). “La investigación sobre comunicación en España (1998-2007). Análisis de los artículos publicados en revistas científicas". Revista latina de comunicación social, v. 66, pp. 101-129.

https://doi.org/10.4185/RLCS-66-2011-926-101-129 
Masip, Pere (2005). "Investigación europea en comunicación durante los años 1994 - 2004: un enfoque bibliométrico". First European communication conference, European Communication Research and Education Association, Amsterdam, 24-26 Nov.

Official journal of the European Union (2013). Regulation No 1291/2013 of the European Parliament and of the Council of 11 December 2013 establishing Horizon 2020 - the Framework Programme for Research and Innovation (2014-2020) and repealing Decision No 1982/2006/EC.

https://ec.europa.eu/research/participants/data/ref/h2020/legal_basis/fp/h2020-eu-establact_en.pdf

Piñuel-Raigada, José-Luis (2009). “La comunicación como objeto científico de estudio, como campo de análisis y como disciplina científica". Contratexto digital, n. 18, pp. 1-22.

https://www.ucm.es/data/cont/docs/268-2013-06-17-Pinuel_ComunicacionOjeto_Campo_Disciplina_Contratexto_2009.pdf

Piñuel-Raigada, José-Luis (2011). La docencia y la investigación universitarias en torno a la comunicación como objeto de estudio en Europa y América Latina. Colección cuadernos artesanos de Latina, n. 15. La Laguna-Tenerife: SLCS.

http://www.cuadernosartesanos.org/067/cuadernos/15_espanol.pdf

Piñuel-Raigada, José-Luis; Caffarel-Serra, Carmen; Lozano-Ascencio, Carlos; Gaitán-Moya, Juan-Antonio (2018). Investigación, comunicación y universidad. Salamanca: Comunicación Social. ISBN: 9788417600129

Piñuel-Raigada, José-Luis; Lozano-Ascencio, Carlos; García-Jiménez, Antonio (eds.) (2011) Investigar la comunicación en España. Vol. I. Universidad Rey Juan Carlos: Fuenlabrada (Madrid).

Piñuel-Raigada, José-Luis; Lozano-Ascencio, Carlos; Gaitán-Moya, Juan-Antonio (2015). "Propuesta de estudio para realizar un mapa de la investigación en comunicación en América Latina". Revista latinoamericana de ciencias de la comunicación (Alaic), v. 12, n. 22 (12), pp. 44-54.

https://www.alaic.org/revista/index.php/alaic/article/view/700

Repiso-Caballero, Rafael; Delgado-López-Cózar, Emilio; Torres-Salinas, Daniel (2011). "Análisis bibliométrico y de redes sociales en tesis doctorales españolas sobre televisión (1976/2007)”. Comunicar, v. XIX, n. 37, pp. 151-159.

https://doi.org/10.3916/C37-2011-03-07

Rodrigo-Alsina, Miquel; García-Jiménez, Leonarda (2010). “Communication theory and research in Spain: A paradigmatic case of a socio-humanistic discipline". European journal of communication, v. 25, n. 3, pp. 273-286.

https://doi.org/10.1177/0267323110373458

Rodríguez-Gómez, Eduardo-Francisco (2016). “Aportación científica de los congresos de la AE-IC (2008-2014): reseña histórica y estado actual de la investigación en comunicación". Historia y comunicación social, v. 21, n. 1, pp. 115-137. https://doi.org/10.5209/rev_HICS.2016.v21.n1.52687

Rodríguez-Serrano, Aarón; Gil-Soldevilla, Samuel (eds.) (2018). Investigar en la era neoliberal. Visiones críticas sobre la investigación en comunicación en España. Barcelona: Aldea Global, UAB, UJI, UPF, UV. ISBN: 9788449080401

Sánchez-de-Madariaga, Inés (ed.) (2011). Libro blanco: Situación de las mujeres en la ciencia española. Unidad de Mujeres y Ciencia, Ministerio de Ciencia e Innovación.

http://www.ciencia.gob.es/stfIs/MICINN/Ministerio/FICHEROS/UMYC/LibroBlanco-Interactivo.pdf

Vasallo, Maria-Immacolata (1999). "La investigación de la comunicación: cuestiones epistemológicas, teóricas y metodológicas”. Diálogos de la comunicación, v. 56, pp. 12-27.

Vidales-Gonzales, Carlos (2015). "Historia, teoría e investigación de la comunicación”. Comunicación y sociedad, v. 22, pp. 11-43.

https://doi.org/10.32870/cys.v0i23.60

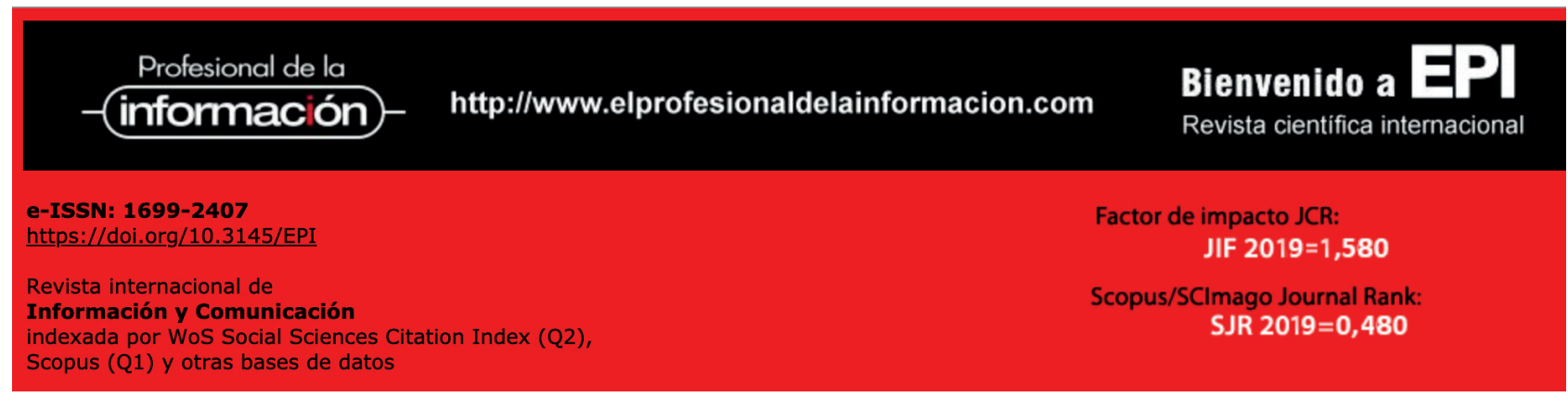

